SHORT REPORT

\title{
Quality and effectiveness of an emergency department during weekends
}

\author{
Ò Miró, M Sánchez, G Espinosa, J Millá
}

Emerg Med J 2004;21:573-574. doi: 10.1136/emj.2002.001420

\begin{abstract}
Objective: To evaluate whether the quality and effectiveness of an emergency department (ED) are modified during weekends. Methods: Quality and effectiveness markers were determined during 539 consecutive days, comparing them according to the day of the week. Quality markers were the daily percentage of patients who died in the internal medical unit (deaths, D), leave ED without being seen (flights, $\mathrm{F}$ ); returned to the ED (revisits, R), and the percentage of registered complaints (C). Effectiveness markers were: the "number of patients waiting to be seen" (WP), the "waiting time to be seen" (WT), and the "length of visit" (LV).

Results: Quality and effectiveness of ED do not worsen during weekend days and some markers significantly improved during such days: $C$ experienced a $26 \%$ decrease $(p=0.001)$, WT decreased 65\% $(p<0.001)$, WP 59\% $(p<0.001)$, and LV 24\% $(p<0.01)$. Assessing the relation between daily number of visits to ED and the quality and effectiveness markers, a significant and direct association was found of the number of visits with $D, F, R$, and WP. Conclusion: Some of the quality and effectiveness markers of the ED improved during weekend days compared with workdays.
\end{abstract}

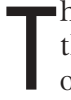
he emergency department (ED) task differs from that of the rest of hospital areas in several aspects. An important one is that EDs exhibit a highly consistent day to day burden of disease. Because of this, EDs (in contrast with the rest of hospital departments) usually maintain a constant amount of human and technical resources throughout the time. The final purpose is to offer to patients attending ED the same level of medical care 24 hours a day and seven days a week without deterioration in quality or effectiveness. However, this notion has not previously been investigated. It could be conceivable that decreases in ED staff expertise or increases in acuity of patients arriving at EDs during weekends imply differences in the final quality and effectiveness of the care provided by EDs. For example, in a recent study, Bell and Redelmeier ${ }^{1}$ have reported increased inhospital mortality during the first 48 hours of hospital stay among patients who were admitted on weekends compared with those admitted on weekdays. These results can eventually be related to a decline in ED performance, because the authors only included in their study acute care admissions from ED and, for many hospitals, ED physicians provide the majority of medical care for such inpatients during weekends. To ascertain or refute this supposition, we tested the null hypothesis that there is no difference in terms of quality and effectiveness of ED between week and weekend days.

\section{METHODS}

This study was performed in a tertiary care teaching hospital, which provides medical care to an urban population of about 500000 people. The ED has 125000 patients a year who are distributed among several units in accordance with their main complaint. The internal medicine unit is divided into three different areas: "sight waiting area" (unlimited capacity), where no real assistance is provided; "first aid area" (13 cubicles), which has three levels of severity, the most important goal is the initial clinical assessment and diagnosis of patients; and "treatment and observation area" ( 33 cubicles), where patients remain until the decision is made whether to discharge or admit. Patients arriving at ED are immediately seen by a triage physician who categorises them according to severity level of their complaint. Human resources of the internal medicine unit are highly constant during the day and the week, and consist of two senior physicians, seven junior physicians (residents), nine nurses, and four clinical assistants. ${ }^{2}$

We determined daily diverse quality and effectiveness markers during 77 consecutive weeks (539 days) and compared them according to the day of the week. As weekend days, we considered all Saturdays, Sundays, and all local holidays (168 eligible days, available data in 166). The remaining days were computed as weekdays (371 eligible days, available data in 370). The total ED attendance was daily compiled, and the mean of daily visits for both weekdays and weekend obtained.

Four quality markers were chosen: deaths, flights, revisits, and complaints. All of them were expressed as a daily percentage with respect to the total ED visits for each day. As "deaths", we included all patients who arrived alive and died in the ED within the first 24 hours. "Flights" were defined as patients who left ED without being seen by a physician or before completing the medical visit. Patients who were discharged and unexpectedly returned to ED within the next 72 hours were considered "revisits". Finally, as "complaints" we counted both those registered on the complaint book located in the ED, and those made at the "user attention unit", whatever the cause of the complaint was.

Three effectiveness markers were analysed. Firstly, the "number of patients waiting to be seen", which was defined as the mean of triaged patients waiting to begin the medical visit. Secondly, the "waiting time to be seen", which was calculated as the mean of the waiting times of the three patients who were waiting to begin the medical visit for longer. Lastly, the "length of visit", which was determined as the elapsed time from medical visit beginning to discharge. In such a marker, the elapsed time of those patients who had to be admitted was not included, as it is usually influenced by several circumstances not always directly related to the ED itself. All these measurements were performed at three hour intervals, at $300,600,900,1200,1500,1800,2100$, and 2400 hours. For all previously defined markers, lower values are associated with higher levels of ED quality or effectiveness.

Abbreviations: $E D$, emergency department; $D$, deaths; $F$, flights; $C$, complaints; WP, patients waiting to be seen; $R$, revisits; WT, waiting time; LV, length of visit 
Results were expressed as mean (SEM). Comparisons among groups were performed using unpaired $t$ test or, alternatively, Mann-Whitney $U$ test if the variable was not distributed according to normality. Linear regression analysis was used to evaluate association between quantitative variables. A p value less than 0.05 were considered significant.

\section{RESULTS}

Daily number of ED attendance was 110 (1) for weekdays and 97 (1) for weekends $(p<0.001)$. There were no differences in severity of illness, measured by the Charlson score for comorbidity, between patients admitted on weekends and those admitted on weekdays. There were no differences in level of seniority and experience between physicians and nurses who work at weekends compared with those who work at weekdays.

We found that quality markers on weekends were either equal to those on weekdays, or even better, just as the percentage of complaints, which experienced a $26 \%$ decrease with respect to weekdays (fig 1).

Regarding effectiveness markers, all of them significantly improved on weekend days compared with weekdays: waiting time decreased by $65 \%$, length of visit by $24 \%$, and triaged patients waiting to begin the medical visit by $59 \%$ (fig 2 ).

When we assessed the relation between quality and effectiveness and the number of daily visits to the ED, we found a significant and direct association between the external pressure supported by the ED (represented by ED census) and the percentage of flights, deaths, and revisits, and number of triaged patients waiting to be seen (table 1).

\section{DISCUSSION}

We have found that quality and effectiveness of our ED do not decrease during weekend days compared with workdays. In fact, most of studied effectiveness and quality markers improved during weekend days and only revisits, although not statistically significant, exhibited a relative increase during weekends. A possible explanation of this trend would be that people have not the opportunity to use their local health centres during weekends.

We believe that, with the same level of human and technical ED resources, quality and effectiveness improvement might be attributed to the significant decline in ED census observed during weekends. This subjective impression is reinforced by the evidence that an increase in the number of daily visits to ED was associated with an increase for many of the markers evaluated by this study or, in other words, with decreased quality and effectiveness. This relation shows the negative influence of staff workload in quality and effectiveness, as it has been shown in intensive care units ${ }^{3}$ and even in EDs. ${ }^{5}$ It is possible that that relation can also be

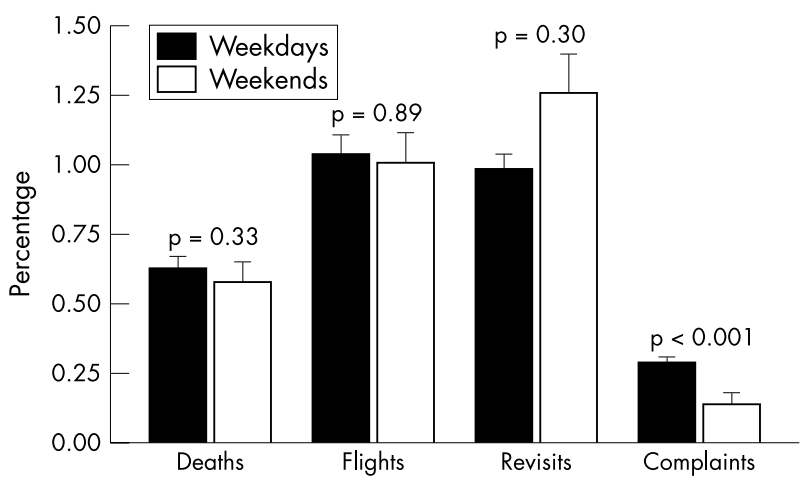

Figure 1 Quality markers.

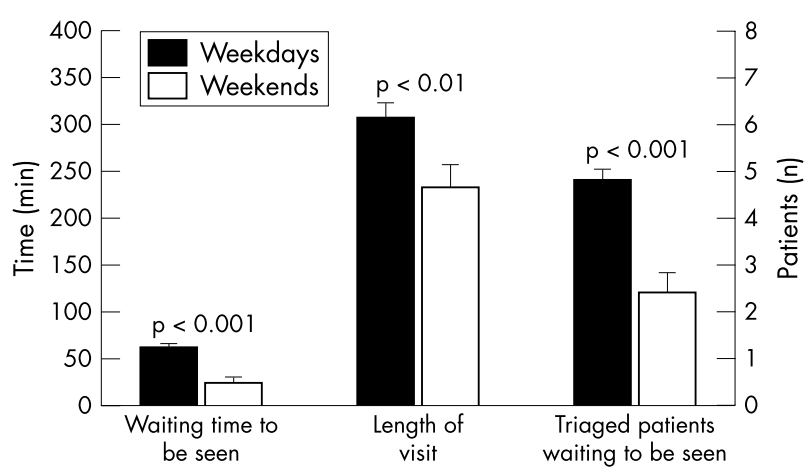

Figure 2 Effectiveness markers.

Table 1 Analysis of association between daily ED census and quality and effectiveness markers

\begin{tabular}{llll}
\hline & Slope & $\boldsymbol{r}$ & $\mathbf{p}$ \\
\hline Quality markers & & & \\
Deaths (\%) & 2.76 & 0.12 & $<0.05$ \\
Flights (\%) & 3.24 & 0.27 & $<0.001$ \\
Revisits (\%) & 1.93 & 0.11 & $<0.05$ \\
Complaints (\%) & 4.43 & 0.08 & 0.17 \\
Effectiveness markers & & & \\
Waiting time to be seen (min) & 0.003 & 0.03 & 0.57 \\
Length of visit (min) & 0.002 & 0.06 & 0.30 \\
Triaged patients waiting to be seen (n) & 0.27 & 0.22 & $<0.001$ \\
\hline & & & \\
\hline
\end{tabular}

extrapolated for patients on general wards. Inhospital wards suffer a dramatic decrease in number and seniority of staff members during weekends, and this increased staff workload can finally lead to a decrease in quality, as shown by Bell and Redelmeier. ${ }^{16}$

In any case, a dynamic matching between human resources and hospital healthcare necessities is mandatory to reach the maximum quality and effectiveness without further deterioration in efficiency.

\section{CONTRIBUTORS}

OM, MS, GE, and JM conceived the study. OM analysed the data. OM, MS, and GE supervised data collection. OM and GE drafted the manuscript, and all authors contributed substantially to its revision.

\section{Authors' affiliations}

Ò Miró, M Sánchez, G Espinosa, J Millá, Emergency Department, Hospital Clínic, Villarroel 170, 08036 Barcelona, Catalonia, Spain

Correspondence to: Dr Ò Miró, Department of Internal Medicine, Hospital Clínic, Villarroel 170, 08036 Barcelona, Catalonia, Spain; omiro@clinic.ub.es

Accepted for publication 25 January 2003

\section{REFERENCES}

1 Bell CM, Redelmeier DA. Mortality among patients admitted to hospitals on weekends as compared with weekdays. N Engl J Med 2001;345:663-8.

2 Miró O, Sánchez M, Coll-Vinent B, et al. Indicadores de calidad en urgencias: comportamiento en relación con la presión asistencial. Med Clin (Barc) $2001 ; 116: 92-7$

3 Tarnow-Mordi WO, Hau C, Warden A, et al. Hospital mortality in relation to staff workload: a 4-year study in an adult intensive-care unit. Lancet 2000;356:185-9.

4 Blunt MC, Burchett KR. Out-of-hours consultant cover and case-mix-adjusted mortality in intensive care. Lancet 2000;356:735-6.

5 Miró O, Sánchez M, Millá J. Hospital mortality and staff workload. Lancet 2000;356:1356-7.

6 Halm EA, Chassin MR. Why do hospital death rates vary? N Engl J Med 2001;345:692-4. 